Gut and Liver, Vol. 13 No. 4, July 2019, pp. 430-439

\title{
Bifidobacterium infantis Induces Protective Colonic PD-L1 and Foxp3 Regulatory T Cells in an Acute Murine Experimental Model of Inflammatory Bowel Disease
}

\author{
Linyan Zhou ${ }^{1}$, Dongyan $\mathrm{Liu}^{2}$, Ying Xie ${ }^{1}$, Xinjie Yao ${ }^{1}$, and Yan $\mathrm{Li}^{1}$ \\ ${ }^{1}$ Department of Gastroenterology, Shengjing Hospital Affiliated to China Medical University, Shenyang, and ${ }^{2}$ Medical Research Center, \\ Shengjing Hospital of China Medical University, Benxi, China
}

Background/Aims: The current study aims to investigate the protective effects of Bifidobacterium infantis on the abnormal immune response to inflammatory bowel disease (IBD) in dextran sodium sulfate (DSS)-induced colitis. Methods: Eight-week-old BALB/c mice were separated into five groups at random (control, DSS, DSS+B9 [B. infantis $\left.1 \times 10^{9} \mathrm{CFU}\right]$, $\mathrm{DSS}+\mathrm{B} 8$ [B. infantis $\left.1 \times 10^{8} \mathrm{CFU}\right]$, and DSS+B7 [B. infantis $\left.1 \times 10^{7} \mathrm{CFU}\right]$ ). Colitis was induced by $5 \%$ DSS ad libitum for 7 days, at which time we assessed weight, the disease activity index (DAI) score, and the histological damage score. The nuclear transcription factor Foxp3 (a marker of Treg cells), cytokines interleukin-10 (IL-10) and transforming growth factor $\beta 1$ (TGF- $\beta 1$ ), and related proteins (programmed cell death ligand 1 [PD-L1] and programmed cell death 1 [PD-1]) were detected by an immunohistochemical method and Western blot. Results: $B$. infantis increased weight, decreased DAI scores and histological damage scores, increased the protein expression of Foxp3 $(p<0.05)$ and cytokines IL-10 and TGF- $\beta 1$ in mouse colon tissue $(p<0.05)$, and increased the expression of PD-L1 in the treatment groups relative to that in the DSS group $(p<0.05)$. The effect of $B$. infantis on Foxp3 and PD-L1 was dose dependent in the treatment groups $(p<0.05)$. PD-L1 was positively correlated with Foxp3, IL10 , and TGF- $\beta 1$. Conclusions: In a mouse model of IBD, $B$. infantis can alleviate intestinal epithelial injury and maintain intestinal immune tolerance and thus may have potential therapeutic value for the treatment of immune damage in IBD. (Gut Liver 2019;13:430-439)

Key Words: Bifidobacterium infantis; Inflammatory bowel disease; Programmed cell death ligand 1; T-Lymphocytes, regulatory

\section{INTRODUCTION}

Inflammatory bowel disease (IBD) is a chronic, recurrent, and nonspecific intestinal disease that includes the diagnoses of ulcerative colitis (UC) and Crohn's disease (CD). The incidence of IBD in the West ranged from 10 to 30 per 100,000 people, accounting for $0.5 \%$ of the total population in $2015 .^{1}$ The annual incidence of newly diagnosed IBD in Asia is 1.37/10 million, and China has the highest rate in Asia, at 3.44/10 million. ${ }^{2}$ In recent years, many scholars have come to believe that an abnormal immunologic response is the main factor involved in the nosogenesis of IBD. Regulatory T cells (Treg cells) play a key role in the immune response. ${ }^{3}$ Foxp3+ Treg cells are a unique subgroup of $\mathrm{CD} 4+\mathrm{CD} 25+\mathrm{T}$ cells that inhibit the reactive effects of $\mathrm{T}$ cells, mainly by secreting cytokines such as transforming growth factor $\beta 1$ (TGF- $\beta 1$ ), interleukin-10 (IL-10), and IL-35 to maintain the body's immune tolerance. Studies have shown that the reduction of Treg cells, the expression of surface molecules, and the impairment of inhibitory function may be related to the occurrence of IBD. ${ }^{4,5}$ Programmed cell death 1 (PD-1; CD279), a costimulator, is expressed on the surface of activated $\mathrm{T}$ cells, $\mathrm{B}$ cells and natural killer cells. Programmed cell death ligand 1 (PD-L1; B7-H1; CD274) acts as an inhibitory signal and plays a negative regulatory role. The PD-1/PD-L1 pathway is closely related to the proliferation of Treg cells; PD-L1 can promote the development and function of Treg cells. ${ }^{6}$ The application of probiotics as a treatment or adjuvant treatment for IBD has recently attracted interest among researchers. Indeed, studies have shown that Bifidobacterium infantis can promote the proliferation of Treg cells and the secretion of IL-10, TGF- $\beta 1$, and other antiinflammatory factors and inhibit the proinflammatory factors interferon- $\gamma$ (IFN- $\gamma$ ), IL-2, and IL-17A. ${ }^{7,8}$ However, no research to

\footnotetext{
Correspondence to: Yan Li

Department of Gastroenterology, Shengjing Hospital Affiliated to China Medical University, Sanhao Street 36, Shenyang 110004, China

Tel: +86-2496615-26111, E-mail: yanli0227@126.com

Received on July 11, 2018. Revised on October 11, 2018. Accepted on October 17, 2018. Published online March 15, 2019

pISSN 1976-2283 eISSN 2005-1212 https://doi.org/10.5009/gnl18316

(a) This is an Open Access article distributed under the terms of the Creative Commons Attribution Non-Commercial License (http://creativecommons.org/licenses/by-nc/4.0) which permits unrestricted non-commercial use, distribution, and reproduction in any medium, provided the original work is properly cited.
} 
date has investigated the effect of $B$. infantis on the expression of the PD-L1 and PD-1 proteins.

The present study was conducted to observe the effects of $B$. infantis on PD-L1 and PD-1, as well as on the Treg cell nuclear transcription factor Foxp3 and the anti-inflammatory factors IL-10 and TGF- $\beta 1$, to understand the effect of $B$. infantis on the abnormal immune response during IBD.

\section{MATERIALS AND METHODS}

\section{Ethical approval}

The current study was approved by the ethics committee of the hospital (approval number: 2017PS353K). Appropriate measures were taken to reduce the discomfort and injury to which the experimental animals were subjected.

\section{Animals}

Forty 8-week-old BALB/c mice, half female and half male, weighing $20 \pm 2$ g, were purchased from Beijing Hua Fu Kang Biological Polytron Technologies, Inc. All feeding and experimental procedures were carried out under specific pathogen-free conditions at the animal experiment center of Shengjing Hospital, China Medical University. The temperature was set between $20^{\circ} \mathrm{C}$ and $26^{\circ} \mathrm{C}$, and the relative humidity was $40 \%$ to $70 \%$. Each animal had free access to food and sterile water.

\section{Reagents}

Dextran sodium sulfate (DSS; molecular weight, 36,000 to 50,000 ) was purchased from MP Biomedicals (Solon, OH, USA). Freeze-dried bacteria powder containing $1.6 \times 10^{11}$ colonyforming units (CFU)/g of $B$. infantis was provided by Shandong Sinovac Biotech Co., Ltd. (Number: 2017012; Beijing, China). We purchased antibodies specific for the following proteins for immunohistochemical and Western blot experiments: PD-L1, PD-1, Foxp3 (Proteintech Group, Inc., Chicago, IL, USA), IL-10, TGF- $\beta 1$, and GAPDH (Abcam, Cambridge, UK). The remaining reagents were obtained from the indicated sources. The Polymer Horseradish Peroxidase Detection System for rabbit primary antibody, the diaminobenzidine (DAB) kit, and PeroxidaseConjugated AffiniPure goat anti-rat IgG were from ZSGB-BIO Co. (Beijing, China), and the enhanced BCA protein assay kit and sodium dodecyl sulfate-polyacrylamide gel electrophoresis (SDS-PAGE) reagents were from Beyotime Institute of Biotechnology (Jiangsu, China).

\section{Experimental design}

Forty mice were randomly divided into five groups: control, DSS, DSS+B9 (treated with $1 \times 10^{9}$ CFU bacteria), DSS+B8 (treated with $1 \times 10^{8}$ CFU bacteria), and DSS+B7 (treated with $1 \times 10^{7} \mathrm{CFU}$ bacteria). The mice in the control group drank water ad libitum for 7 days, while the mice in the other four groups were given 5\% DSS dissolved in drinking water and allowed to drink ad libi- tum for 7 days. The control and DSS groups were administered $400 \mu \mathrm{L}$ of normal saline (NS) daily intragastrically, while the other treatment groups were given $400 \mu \mathrm{L}$ of NS $+B$. infantis at the corresponding concentration via the same procedure. Body weight, fecal characteristics, and occult blood test results were recorded each day. Disease activity index (DAI) scores were calculated according to the Hamamoto et al. (Table 1). ${ }^{9}$ After 7 days of colitis induction, the mice were euthanized by inhalation of isoflurane anesthesia, and one-fourth of the colon tissue near the anus was fixed with 4\% paraformaldehyde, while the rest of the colon tissues were immediately cryopreserved at $-80^{\circ} \mathrm{C}$ for further analysis.

\section{Histologic analysis}

After fixation with 4\% paraformaldehyde for 48 hours, the colonic tissues were routinely dehydrated, paraffin embedded, sliced at a 4- $\mu \mathrm{m}$ thickness, and stained with hematoxylin and eosin. Each slice was subjected to double-blind histological damage scoring of three different randomly selected highmagnification visual fields (400x) using the Dieleman integral standard (Table 2). ${ }^{10}$

\section{Immunohistochemistry}

Nuclear transcription factor FOXP3+ Treg cells, the cytokines IL-10 and TGF- $\beta 1$, and the related proteins PD-L1 and PD- 1 were detected using immunohistochemical staining, and 4- $\mu \mathrm{m}-$ thick slices were dewaxed with xylene and an alcohol gradient. The slices were then repaired using antigen retrieval in a microwave for 7 minutes. After naturally cooling to room temperature, the sections were incubated in peroxidase for 10 minutes and blocked with serum for 15 minutes, followed by overnight incubation $\left(4^{\circ} \mathrm{C}\right)$ with a rabbit antibody (Foxp3, dilution 1:8,000; IL-10, dilution 1:600; TGF- $\beta 1$, dilution 1:700; PD-1, dilution 1:8,000; and PD-L1, dilution 1:800). In the negative control group, phosphate-buffered saline (PBS) was used to replace the first antibody. The slides were incubated with secondary antibody for 30 minutes $\left(37^{\circ} \mathrm{C}\right)$, then incubated with horseradishlabeled streptomycin for 15 minutes, and DAB staining was used to increase the background color. The sections were stained with hematoxylin, dehydrated with graded ethanol and xylene, and sealed with neutral gum. After incubation with all reagents except for serum, the sections were soaked three times in PBS

Table 1. Scoring of the Disease Activity Index

\begin{tabular}{ccll}
\hline Score & $\begin{array}{c}\text { Weight } \\
\text { loss }(\%)\end{array}$ & $\begin{array}{c}\text { Stool } \\
\text { consistency }\end{array}$ & $\begin{array}{c}\text { Visible blood } \\
\text { in feces }\end{array}$ \\
\hline 0 & None & Normal & None \\
1 & $1-5$ & & \\
2 & $6-10$ & Loose & Slight bleeding \\
3 & $11-20$ & & \\
4 & $>20$ & Diarrhea & Gross bleeding \\
\hline
\end{tabular}


Table 2. Scoring System to Calculate Histological Damage

\begin{tabular}{|c|c|c|c|c|}
\hline Score & Inflammation & Depth of lesions & Destruction of crypt & Width of lesions $(\%)$ \\
\hline 0 & None & None & None & - \\
\hline 1 & Slight & Mucosa & 1/3 Basal crypt & $1-25$ \\
\hline 2 & Moderate & Mucosa and submucosa & 2/3 Basal crypt & $26-50$ \\
\hline 3 & Severe & Transmural & Intact epithelium only & $51-75$ \\
\hline 4 & - & - & Total crypt and epithelium & $76-100$ \\
\hline
\end{tabular}

for 5 minutes each. Positive results were indicated by brown granules that appeared after DAB staining. Every slice was examined, and three fields of view were randomly selected. Image acquisition was conducted with a Nikon E800 microscope, and semiquantitative detection of optical density was conducted with NIS-Elements Br3.0 software (Nikon Instruments, Inc., Tokyo, Japan).

\section{Western blot analysis}

Colonic tissues were lysed in RIPA buffer with PMSF, and the protein concentration of the colonic tissue lysate was detected after chopping and homogenizing the tissue on ice. A 12\% trisglycine gel was used, and the proteins were transferred to a polyvinylidene difluoride (PVDF) membrane after constant pressure electrophoresis (Millipore Corp., Billerica, MA, USA). The membrane was blocked with 2.5\% bovine serum albumin and incubated overnight $\left(4^{\circ} \mathrm{C}\right)$ with antibodies specific for Foxp3 (dilution of $1: 1,000$ ), IL-10 (dilution of $1: 800$ ), TGF- $\beta 1$ (dilution of 1:500), PD-L1 (dilution of 1:750), PD-1 (dilution of 1:500) and GAPDH (dilution of 1:10,000). Goat horseradish peroxidase linked to an anti-rabbit IgG secondary antibody was added at a dilution of 1:5,000 and incubated for 1.5 hours at room temperature. Antibody-specific bands were detected using BioImaging Systems (Amersham Imager 600; GE Healthcare Life Sciences, Pittsburgh, PA, USA).

\section{Statistical analysis}

All statistical data were analyzed by GraphPad Prism 7.0 (GraphPad Software, La Jolla, CA, USA) and SPSS version 23.0 (IBM Corp., Armonk, NY, USA). The measurement data were expressed as the mean \pm standard deviation. Two-way analysis of variance (ANOVA) was used to examine data related to body weight and DAI scores. For the remaining data, all data that had a normal distribution were analyzed using one-way ANOVA, and all data that were abnormally distributed were analyzed using the Friedman test. Spearman correlation demonstrated significant correlations between PD-L1 and Foxp3, IL-10, and TGF- $\beta 1$ (all $\mathrm{p}<0.05$ ).

\section{RESULTS}

\section{General condition and body mass changes of the mice in each group}

The mice in the control group were generally in good condition, active, and sensitive to stimuli, with normal eating, no diarrhea or bloody stools, and smooth hair color, and while some mice exhibited weight loss, the overall trend was an increase in weight. In contrast, mice exposed to DSS showed signs of mental deterioration, decreased activity, loose and bloody stool, dry hair, and weight loss. The weight loss of the DSS group was significant compared to that of the control group after 4 days $(p<0.01)$. Compared to that of the DSS group, the weight of the DSS+B9 group from days 5 to 7 , the weight of the DSS+B8 group from days 6 to 7, and the weight of the DSS+B7 group on day 7 trended upward $(\mathrm{p}<0.05)$; however, these three groups did not differ significantly from one another in terms of weight (Fig. 1A). On the 7th day after model induction, the weight loss of the mice in the control, DSS, DSS+B9, DSS+B8 and DSS+B7 groups were as follows: $1.31 \pm 0.26 \mathrm{~g},-3.44 \pm 0.62 \mathrm{~g},-1.31 \pm 0.46 \mathrm{~g}$, $-1.75 \pm 0.27 \mathrm{~g}$, and $-1.94 \pm 0.42 \mathrm{~g}$, respectively.

\section{DAl scores}

The DAI score in the control group ranged from 0 to $0.25 \pm 0.71$. On the $3 r d$ day, the test group began to appear thin, had loose, bloody stool and showed signs of other disease activity, and the DAI scores increased until they were significantly different from those of the control group $(\mathrm{p}<0.05)$. The DAI scores for the DSS, DSS+B9, DSS+B8, and DSS+B7 groups peaked on day 7 , with values of $7 \pm 0.76,3.38 \pm 0.52,4.63 \pm 0.52$, and $5.5 \pm 0.53$, respectively. On the 5 th day, the DAI scores of the $B$. infantis groups were significantly lower than those of the DSS group, and the DAI scores were significantly different among the groups treated with different concentrations on day 7 (all $\mathrm{p}<0.05$ ) (Fig. 1B).

\section{Histologic damage}

To investigate the effect of $B$. infantis on disease progression in mice with colitis, histological analysis of the colon was performed. Compared to those of the mice in control group, the colons of the mice in the DSS group were congested, shortened, and thickened, with visible hemorrhaging. In the DSS+B9, 

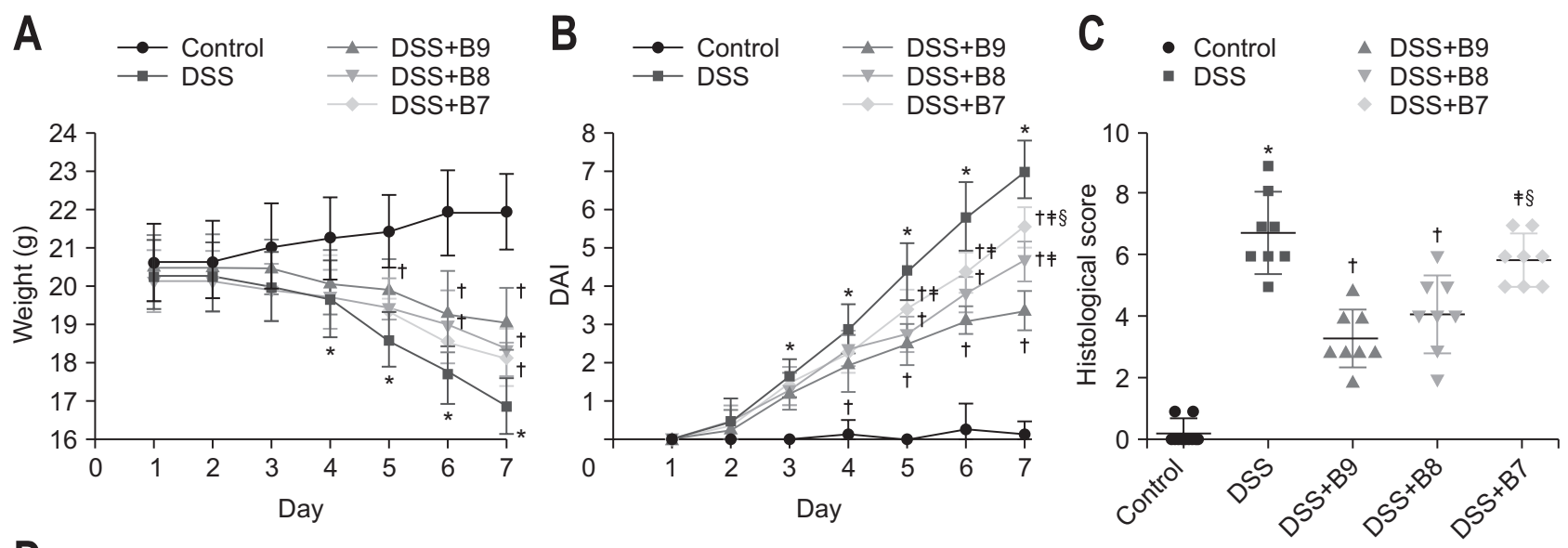

D

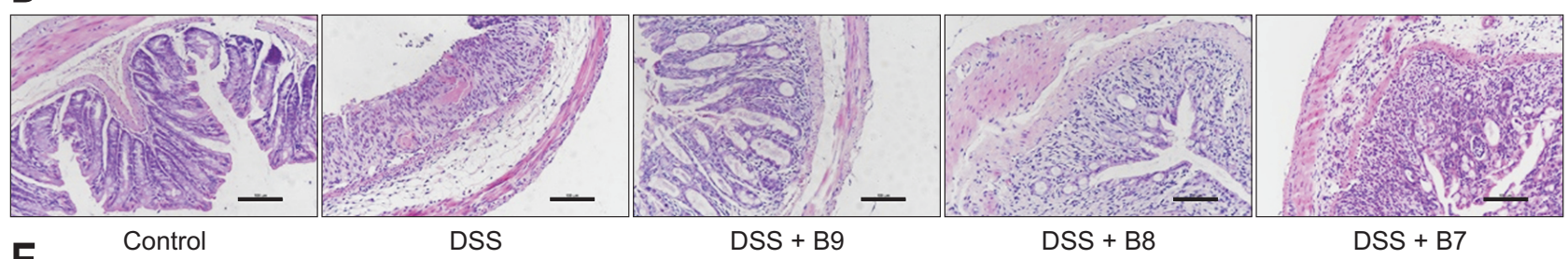

$\mathrm{E}$

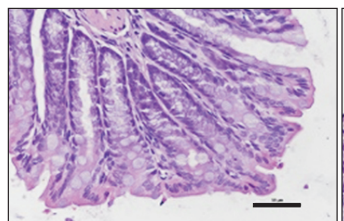

Control

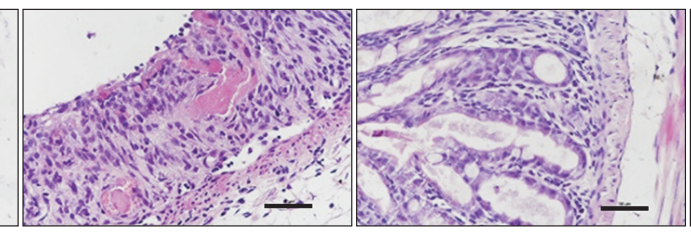

DSS

DSS + B9

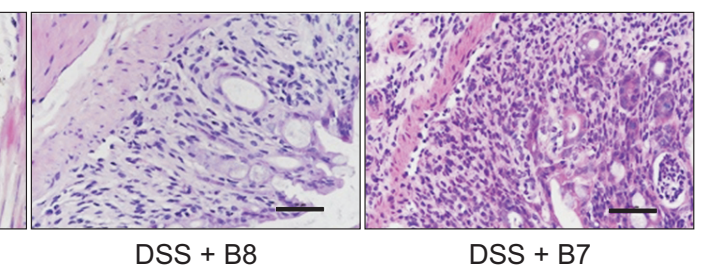

Fig. 1. (A) Effect of Bifidobacterium infantis on weight. (B) Disease activity index (DAI) changes among groups. Values given as the mean \pm SD ( ${ }^{*} \mathrm{p}<0.01$, compared to the control group; ${ }^{\dagger} \mathrm{p}<0.05$, compared to the dextran sodium sulfate [DSS] group; ${ }^{\dagger} \mathrm{p}<0.05$, compared to the DSS+B9 group; ${ }^{8} \mathrm{p}<0.05$, compared to the DSS+B8 group). (C) Colonic damage scores. Values given as the mean $\pm \mathrm{SD}$ ( $\mathrm{p}<<0.01$, compared to the control group; ${ }^{\dagger} \mathrm{p}<0.01$, compared to the DSS group; ${ }^{\dagger} \mathrm{p}<0.01$, compared to the DSS+B9 group; ${ }^{8} \mathrm{p}<0.05$, compared to the DSS+B8 group). (D) Representative H\&Estained colon sections are shown (original magnification, $\times 200$ ). Control group: normal crypt architecture and absence of inflammation; DSS group: mucosal structure was damaged, extensive crypts were destroyed, and large numbers of infiltrating inflammatory cells; DSS+B9 group: mild inflammation involved the mucosal layer, and the crypt damage was less than 1/3; DSS+B8 group: mild inflammation involved the submucosa, and the crypt damage was between $1 / 3$ and 2/3; DSS+B7 group: mild inflammation involved the muscle layer, and the crypt damage was 2/3. (E) Histopathology of the colon, representative H\&E-stained colon sections (original magnification, $\times 400$ ).

DSS+B8, and DSS+B7 groups, the edema and intestinal wall thickness of the mice were reduced compared to those of the mice treated with DSS, and no bleeding was observed. Hematoxylin and eosin staining showed that the colons of the mice in the control group had an ordered arrangement, with normal crypts and almost no inflammatory cell infiltration. In the DSS group, the mucosal structure was damaged, characterized by disordered adenoid tube arrangement, extensive deformations and atrophy of recesses, decreased goblet cells, atrophy and necrosis of the epithelium, disappearance of epithelial cells, and infiltration of inflammatory cells (involving the lamina propria and submucosa), with recesses and cryptic abscesses. The histological injury score of the DSS group was clearly higher than that of the control group $(p<0.01)$, and some of the specimens showed evidence of ulceration. The DSS+B9 group showed mild inflammation that involved the mucosal layer. The scope of the damaged crypts was less than one-third of the crypts. The epi- thelium was complete, and the width of the lesions comprised less than 25\% of the epithelium. The DSS+B8 group had mild inflammation, partially involving the submucosa, and the crypt damage ranged from one-third to two-thirds of the crypts, with the width of the lesions comprising less than 50\% of the epithelium. The DSS+B7 group had moderate inflammation, partially involving the submucosal layer, two-thirds of the crypts were damaged, with the width of lesions ranging from 50\% to 75\% of the epithelium. Compared to that in the DSS+B7 group, the histological injury score in the DSS+B9 group was significantly increased, but there was no striking difference between the DSS+B8 and DSS+B9 groups (Fig. 1C-E).

\section{The effect of $B$. infantis on Treg cells}

To study the effect of $B$. infantis on immune function in mice with colitis, we examined Treg cells. Foxp3 is a nuclear transcription factor of Treg cells. Immunohistochemistry showed 
that brown granules were deposited in the lymphocytes of the lamina propria. Compared to those in the control group, the protein expression, number of cells with positive staining, and staining intensity of Foxp3 in the DSS group were significantly reduced $(p<0.01)$. Similarly, compared to those in the DSS group, the number and intensity of cells with positive staining for Foxp3 were significantly increased $(\mathrm{p}<0.05)$, and differences were observed among the groups treated with different concentrations of $B$. infantis $(\mathrm{p}<0.05)$. Additionally, the protein expression of Foxp3 was significantly decreased in the DSS group compared to that in the control group $(\mathrm{p}<0.01)$. The expression of Foxp3 in the groups treated with B. infantis was significantly increased $(\mathrm{p}<0.05)$ compared to that in the DSS group, and an expression difference was found among the groups treated with different concentrations of $B$. infantis $(\mathrm{p}<0.05)$ (Fig. 2).

\section{B. infantis promoted the expression of IL-10 and TGF- $\beta 1$}

IL-10 and TGF- $\beta 1$ are secretory proteins that are widely expressed in mucous membranes and the lamina propria. The expression of the IL-10 protein in the DSS group was conspicuously lower than that in the control group. The expression of $\mathrm{IL}-10$ in all the $B$. infantis groups was higher than that in the DSS group. The IL-10 expression in the DSS+B9 group was higher than that in the DSS+B8 and DSS+B7 groups, but there was no difference between the DSS+B8 and DSS+B7 groups. The protein expression of TGF- $\beta 1$ in the DSS group was significantly lower than that in the control group, and the expression level increased significantly after treatment with $B$. infantis.
The expression of TGF- $\beta 1$ in the DSS+B9 group was significantly higher than that in the DSS+B7 group, and there was a significant difference between the DSS+B9 and DSS+B8 groups in Western blot experiments but not in immunohistochemistry experiments (Fig. 3).

\section{The effect of B. infantis on PD-L1 and PD-1}

PD-L1 was found in the cell membrane and cytoplasm and was expressed in the intestinal epithelium and the lamina propria; PD-1 was also found in the cell membrane and cytoplasm but was observed only in the lamina propria and not the intestinal epithelium. The expression of PD-L1 and PD-1 was high in the control group, and the expression of these factors in the DSS group was significantly lower $(\mathrm{p}<0.01)$. The expression of PD-L1 and PD-1 in the groups treated with $B$. infantis was higher than that in the DSS group $(\mathrm{p}<0.05)$, and there were significant differences in the expression of PD-L1 among the groups treated with different concentrations of $B$. infantis $(p<0.05)$; however, there was no difference in the expression of PD- 1 among the $B$. infantis groups (Fig. 4).

\section{Correlation analysis of PD-L1 with the Foxp3, IL-10, and TGF- $\beta 1$ proteins}

Analysis of protein expression by Western blotting revealed positive correlations of PD-L1 with Foxp3, IL-10, and TGF- $\beta 1$ (all p-values <0.05; r=0.737 [Foxp], r=0.664 [IL-10], and $r=0.722$ [TGF- $\beta 1]$ ) (Fig. 5).
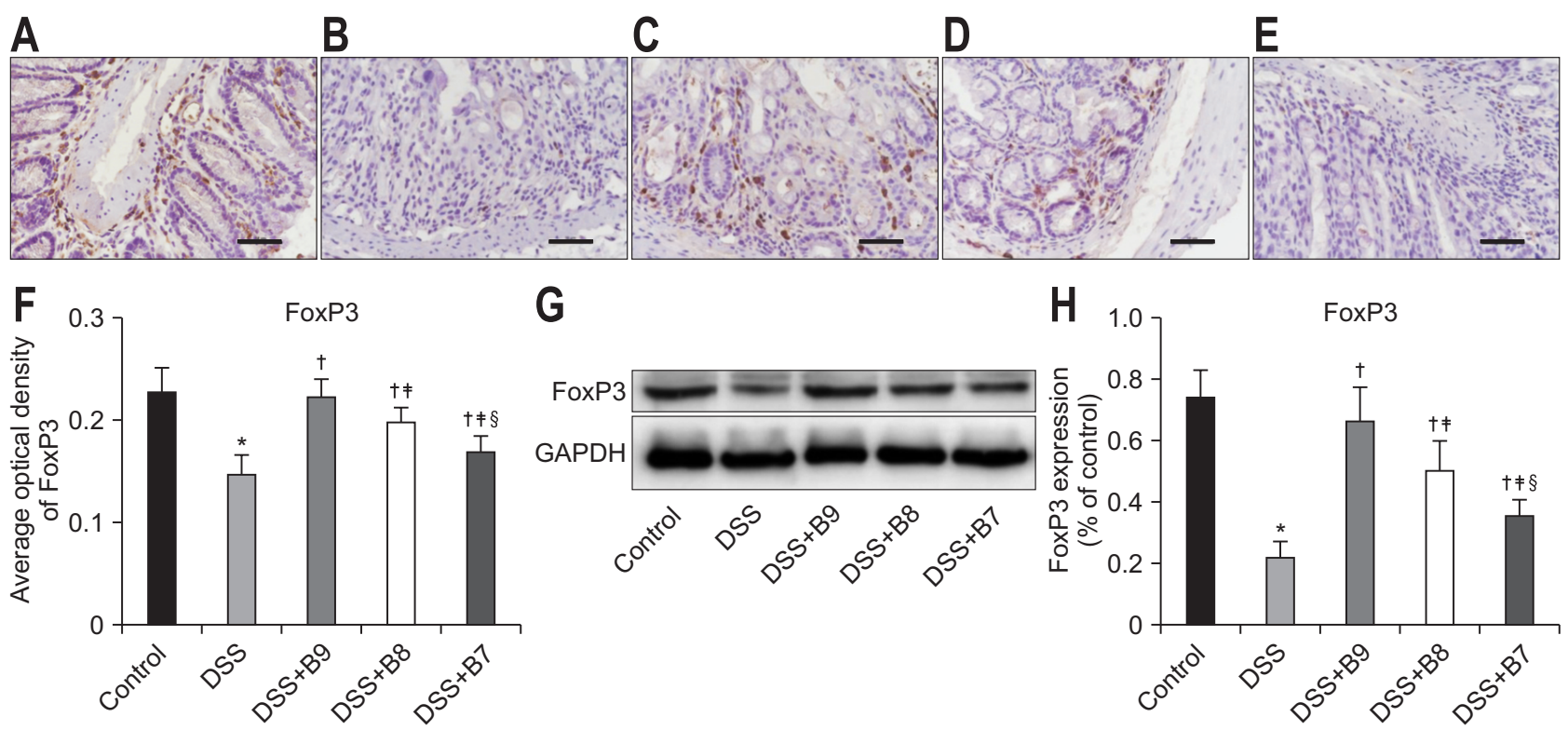

Fig. 2. Effects of Bifidobacterium infantis on Foxp3. (A-E) Foxp3 was detected by immunohistochemistry in the control, dextran sodium sulfate (DSS), DSS+B9, DSS+B8, and DSS+B7 groups (original magnification, $\times 400$ ). (F) Analysis of Foxp3 expression by average optical density according to (A-E). (G) Foxp3 expression in each group by Western blot. (H) Analysis of Foxp3 according to (G). Values given as the mean \pm SD ( ${ }^{*} \mathrm{p}<0.01$, compared to the control group; ${ }^{\dagger} \mathrm{p}<0.05$, compared to the DSS group; ${ }^{\dagger} \mathrm{p}<0.05$, compared to the DSS+B9 group; ${ }^{\circledR} \mathrm{p}<0.05$, compared to the DSS+B8 group). 

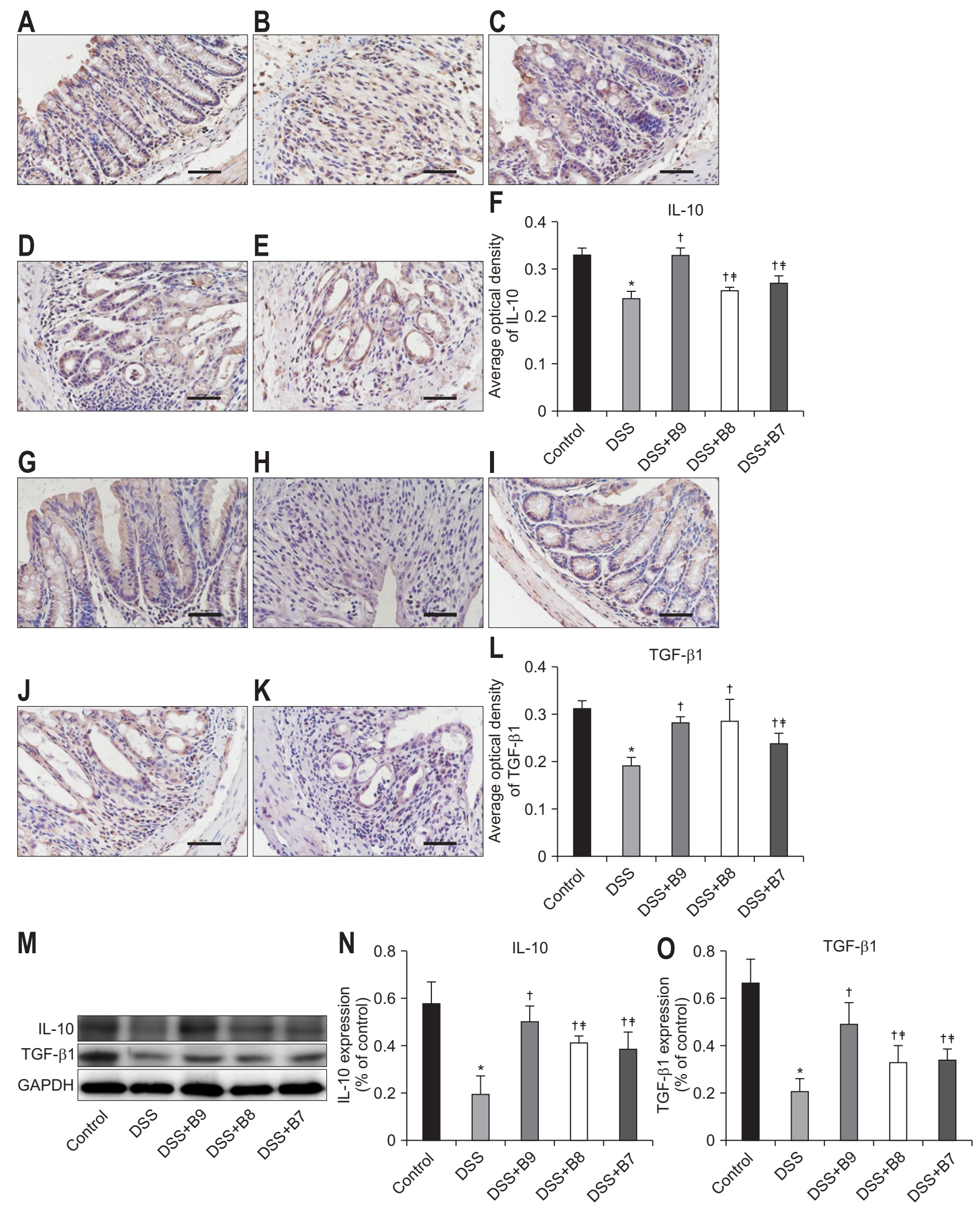

Fig. 3. Effects of Bifidobacterium infantis on interleukin-10 (IL-10) and transforming growth factor $\beta 1$ (TGF- $\beta 1$ ). (A-E) IL-10 and (G-K) TGF- $\beta 1$ were detected by immunohistochemistry in the control, dextran sodium sulfate (DSS), DSS+B9, DSS+B8, and DSS+B7 groups (original magnification, $\times 400$ ). (F, L) Analysis of the expression of IL-10 and TGF- $\beta 1$ by average optical density according to (A-E) and (G-K). (M) Expression of IL10 and TGF- $\beta 1$ in each group by Western blot. (N, 0) Analysis of IL-10 and TGF- $\beta 1$ according to (M). Values given as the mean \pm SD ( ${ }^{*} \mathrm{p}<0.01$, compared to the control group; ${ }^{\dagger} \mathrm{p}<0.05$, compared to the DSS group; ${ }^{\ddagger} \mathrm{p}<0.05$, compared to the DSS+B9 group; ${ }^{8} \mathrm{p}<0.05$, compared to the DSS+B8 group). 

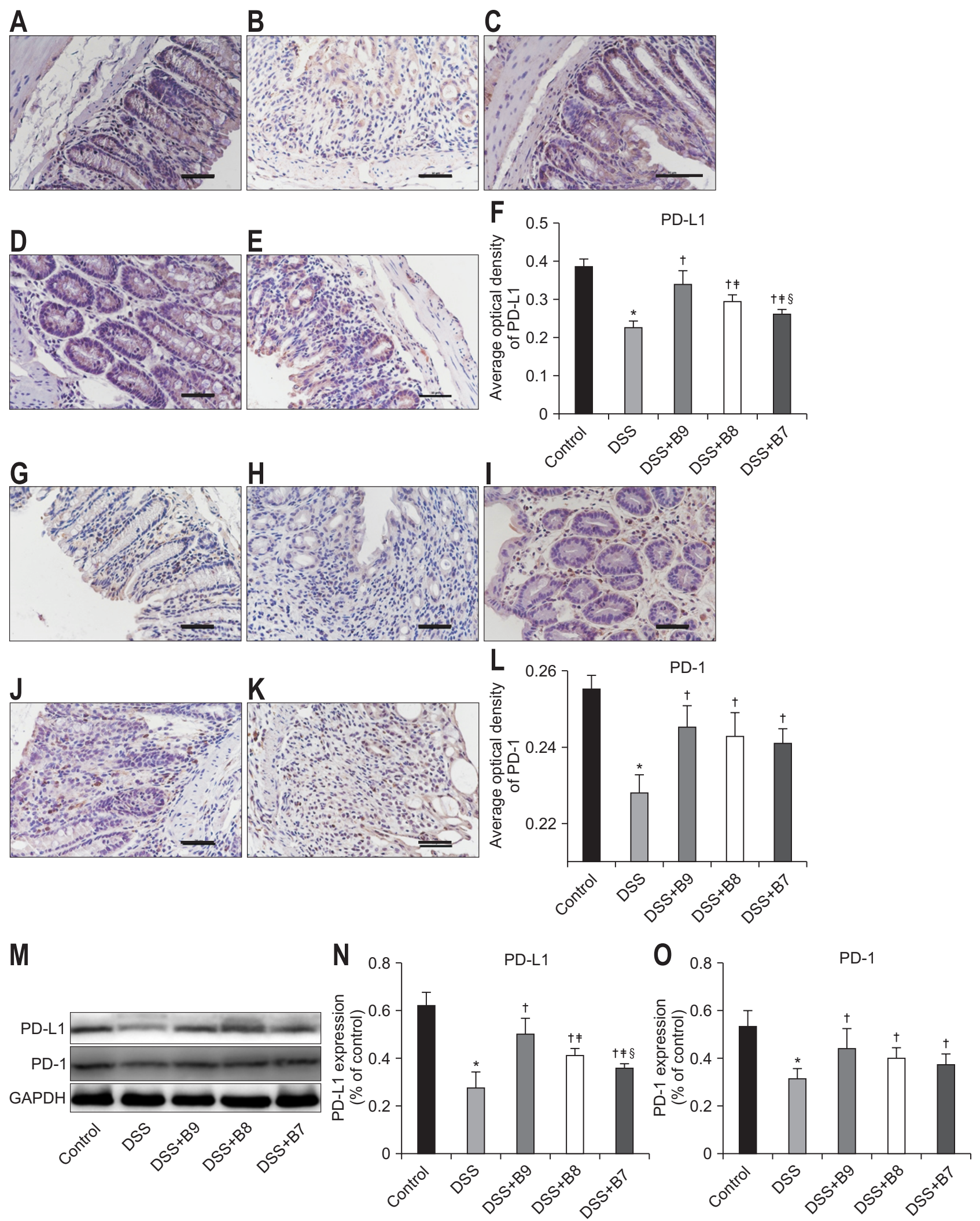

Fig. 4. Effects of Bifidobacterium infantis on PD-L1 and PD-1. (A-E) PD-L1 and (G-K) PD-1 were detected by immunohistochemistry in the control, dextran sodium sulfate (DSS), DSS+B9, DSS+B8, and DSS+B7 groups (original magnification, $\times 400$ ). (F, L) Analysis of the expression of PDL1 and PD-1 by average optical density according to (A-E) and (G-K). (M) Expression of PD-L1 and PD-1 in each group by Western blot. (N, 0) Analysis of IL-10 and TGF- $\beta 1$ according to (M). Values given as the mean \pm SD $\left({ }^{*} \mathrm{p}<0.01\right.$, compared to the control group; ${ }^{\dagger} \mathrm{p}<0.05$, compared to the DSS group; ${ }^{\ddagger} \mathrm{p}<0.05$, compared to the DSS+B9 group; ${ }^{\mathrm{s}} \mathrm{p}<0.05$, compared to the DSS+B8 group).

PD-L1, programmed cell death ligand 1; PD-1, programmed cell death 1. 


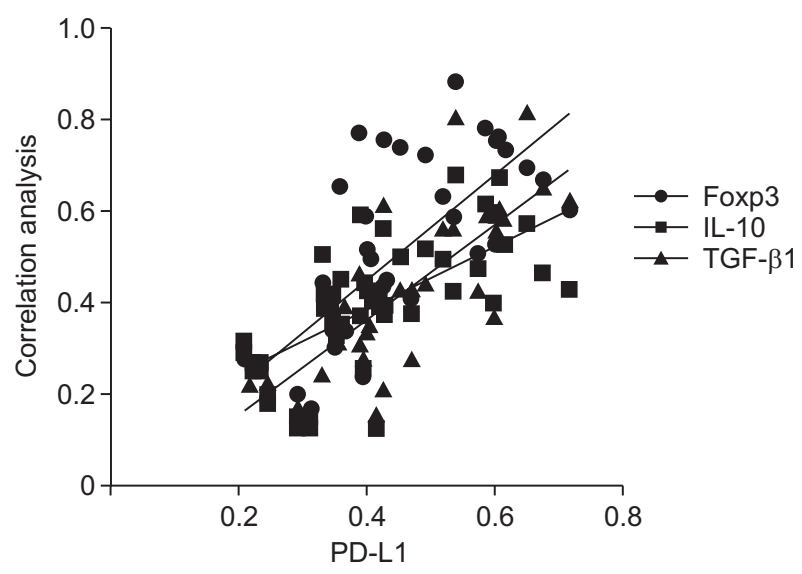

Fig. 5. Correlation analysis between PD-L1 and Foxp3, IL-10, TGF- $\beta 1$ (all $\mathrm{p}<0.05$ ). $\mathrm{r}=0.737$ (Foxp3), $\mathrm{r}=0.664$ (IL-10), $\mathrm{r}=0.722$ (TGF- $\beta 1$ ). PD-1, programmed cell death 1; IL-10, interleukin-10; TGF, transforming growth factor.

\section{DISCUSSION}

Although the diagnosis and treatment of IBD have greatly improved, ${ }^{11}$ the etiology and pathogenesis of IBD are still unclear. The most recent studies suggest that the main pathogenic mechanism of IBD may involve a disruption of the delicate balance between the host and intestinal microbes, which triggers an inflammatory immune response in genetically susceptible individuals. $^{12}$ The histological changes observed in the DSSinduced colitis animal model are very similar to those observed in human IBD. ${ }^{13,14}$ After induction with the DSS solution, the intestinal flora of the experimental animals changes, yielding abnormal immune responses. ${ }^{15}$ Therefore, the DSS-induced colitis animal model is widely used in experimental IBD research. Bifidobacterium is the most important physiological group in the human large intestine. Organisms in this group can inhibit abnormal autoimmune reactions and maintain homeostasis of the intestinal environment. More than 30 species of Bifidobacterium have been identified to date. Of these species, $B$. infantis is the most closely related to human health. Many studies have shown that $B$. infantis has a positive effect on intestinal flora imbalances, acute and chronic diarrhea, irritable bowel syndrome, IBD, and other diseases; ${ }^{16,17}$ however, the immunomodulatory effect and mechanism of IBD need to be further studied. To study the therapeutic effect of $B$. infantis on IBD and its immune mechanism, we conducted animal experiments in an acute colitis model induced by treating mice with DSS with an additional $B$. infantis intragastric intervention. We observed that $B$. infantis can increase the weight of DSS-treated mice and improve symptoms, suggesting that $B$. infantis can delay the acute stage of colitis in mice. In addition, $B$. infantis can improve histological damage scores, and we demonstrated an improvement in histology in the high-concentration treatment group compared to that in the low-concentration treatment group. This finding suggests that $B$. infantis may be able to repair acute intestinal mucosal injury and slow the progression of acute colitis. Therefore, $B$. infantis demonstrated protective effects in mice with acute colitis. Treg cells are a relatively independent subgroup of CD4+ T cells. These cells play an important role in maintaining autoimmune tolerance, regulating immune responses, responding to external antigens, and preventing graft rejection. ${ }^{18}$ Treg cells are involved in the pathogenesis, development, and healing of IBD. ${ }^{19,20}$ Animal experiments have shown that following the injection of CD4+CD25+ Treg cells, the symptoms of enteritis in mice lacking $\mathrm{CD} 4+\mathrm{CD} 25+$ Treg cells were effectively alleviated, suggesting that Treg cells play a crucial role in maintaining intestinal immunological tolerance. ${ }^{21}$ Indeed, clinical studies have shown that the number of Treg cells in the intestinal mucosa of IBD patients is relatively low and correlates with disease activity. ${ }^{4}$ Additionally, Canavan et al. ${ }^{22}$ demonstrated that Treg cells can inhibit lymphocyte function in CD patients, suggesting that Treg cells are involved in autoimmune repair during IBD. Foxp3 is a specific transcription factor involved in the immunosuppression function of Treg cells; high expression can promote Treg cell differentiation and downregulate the immune response. ${ }^{23}$ Treg cells mainly secrete the cytokines IL-10 and TGF- $\beta 1$, which play an immunosuppressive role and help maintain autoimmune tolerance. ${ }^{24}$ IL-10 gene-deficient mice develop spontaneous enteritis, which is characterized by the dysregulation of anti-inflammatory cytokines. ${ }^{25}$ In a preclinical study, the subcutaneous injection of recombinant human IL-10 was found to have a positive effect on IBD patients. ${ }^{26}$ TGF- $\beta 1$ is a multifunctional cytokine that participates in wound repair, embryonic development, cell growth and differentiation, extracellular matrix synthesis, immune regulation, and other processes. ${ }^{27}$ Chowdhury et $a{ }^{28}{ }^{28}$ found that the expression of TGF- $\beta 1$ mRNA in chronic persistent UC patients was lower than that in patients who did not have a UC diagnosis, suggesting that this factor may be a main cause of the chronicity of the disease. Several experiments have proved that Bifidobacterium can promote the proliferation of Treg cells, improve the expression of Foxp3, and enhance immunosuppressive function by enhancing the expression of anti-inflammatory factors, such as IL-10 and TGF- $\beta 1$, thus reducing the immune response of IBD. ${ }^{29,30}$ Indeed, we confirmed that $B$. infantis can increase the protein expression of Foxp3 in the colon tissue of mice in a concentration-dependent manner, suggesting that $B$. infantis may promote the proliferation of Treg cells. The results of studies performed Zheng et al. ${ }^{31}$ suggested that $B$. infantis could improve the expression of Foxp3 in colon tissue, which we also confirmed. We also observed that $B$. infantis enhanced the expression of IL-10 and TGF- $\beta 1$ in the colon, suggesting that $B$. infantis promotes the immunosuppressive function of the colon in mice. Therefore, we speculate that $B$. infantis could play a role in the treatment of IBD by promoting the proliferation and immunosuppressive function of Treg cells and by participating in immune regulation in the intestine. 
PD-L1 combines with its receptor PD-1 to induce immunological tolerance by restraining the activity of $\mathrm{T}$ and $\mathrm{B}$ cells. ${ }^{32}$ In the current study, we found that the expression of PD-L1 and PD-1 in the intestines of mice with acute enteritis decreased significantly, suggesting that PD-L1 and PD-1 may be involved in the pathogenesis of DSS-induced acute enteritis. We also found that $B$. infantis can increase the expression level of PD-L1 and PD-1 in the intestine; however, it is not clear whether $B$. infantis can increase the expression of PD-L1 and PD-1 or if $B$. infantis indirectly stimulates the expression of PD- 1 by increasing the expression of PD-L1. Future studies are needed to answer this question. The animal experiments showed that PD-L1 alleviated the inflammatory response in mice with DSS-induced IBD. ${ }^{33}$ This finding suggests that PD-L1 is involved in the treatment of IBD. Indeed, we demonstrated positive correlations between PD-L1 and Foxp3, IL-10, and TGF $\beta 1$. Whether PD-L1 affected Foxp3, IL-10, and TGF $\beta 1$ or whether these protein levels changed in parallel remains to be explored. Moreover, the expression of PD-L1 was significantly correlated with the number of Foxp3+ Treg cells, suggesting that PD-L1 may be closely related to the proliferation of Treg cells. ${ }^{34,35}$ PD-L1 also plays an important role in inducing the differentiation of Treg cells. ${ }^{36}$ Therefore, we propose that $B$. infantis may increase the expression of PD-L1, further promoting the proliferation and differentiation of Treg cells and enhancing the immunosuppressive function of Treg cells.

In summary, $B$. infantis can increase the expression of the PD-L1 and PD-1 proteins and promote the expression of the Treg cell nuclear transcription factor Foxp3 and the antiinflammatory factors IL-10 and TGF- $\beta 1$. $B$. infantis can also alleviate intestinal epithelial injury, maintain intestinal immune tolerance, and protect mice against IBD. Treatment with $B$. infantis represents a potential therapy for individuals with IBD. The protective role of Treg cells in IBD and the role of PD-L1 induced by $B$. infantis require further investigation.

\section{CONFLICTS OF INTEREST}

No potential conflict of interest relevant to this article was reported.

\section{AUTHOR CONTRIBUTIONS}

L.Z. wrote manuscript, processed the samples, analyzed the raw data, and approved the final version of the manuscript; D.L. critically revised the manuscript and approved its final version: Y.X and X.Y. processed the samples, assessed and scored colon histological damage and approved the final version of the manuscript; Y.L. conceived the study protocol, critically revised the manuscript, obtained funding and approved its final version.

\section{ORCID}

Linyan Zhou

https://orcid.org/0000-0002-9403-2809

Dongyan Liu

https://orcid.org/0000-0002-3902-9316

Ying Xie

Xinjie Yao

https://orcid.org/0000-0001-8226-4242

https://orcid.org/0000-0003-0658-7773

Yan Li

https://orcid.org/0000-0002-5155-6290

\section{REFERENCES}

1. Kaplan GG. The global burden of IBD: from 2015 to 2025. Nat Rev Gastroenterol Hepatol 2015;12:720-727.

2. Ng SC, Tang W, Ching JY, et al. Incidence and phenotype of inflammatory bowel disease based on results from the Asiapacific Crohn's and colitis epidemiology study. Gastroenterology 2013;145:158-165.

3. Sarrabayrouse G, Alameddine J, Altare F, Jotereau F. Microbiotaspecific CD4CD8 alphaplaha Tregs: role in intestinal immune homeostasis and implications for IBD. Front Immunol 2015;6:522.

4. Maul J, Loddenkemper C, Mundt P, et al. Peripheral and intestinal regulatory $\mathrm{CD} 4+\mathrm{CD} 25$ (high) $\mathrm{T}$ cells in inflammatory bowel disease. Gastroenterology 2005;128:1868-1878.

5. Kryczek I, Wang L, Wu K, et al. Inflammatory regulatory T cells in the microenvironments of ulcerative colitis and colon carcinoma. Oncoimmunology 2016;5:e1105430.

6. Tripathi S, Guleria I. Role of PD1/PDL1 pathway, and TH17 and Treg cells in maternal tolerance to the fetus. Biomed J 2015;38:2531.

7. Zuo L, Yuan KT, Yu L, Meng QH, Chung PC, Yang DH. Bifidobacterium infantis attenuates colitis by regulating $\mathrm{T}$ cell subset responses. World J Gastroenterol 2014;20:18316-18329.

8. Fu L, Song J, Wang C, Fu S, Wang Y. Bifidobacterium infantis potentially alleviates shrimp tropomyosin-induced allergy by tolerogenic dendritic cell-dependent induction of regulatory $\mathrm{T}$ cells and alterations in gut microbiota. Front Immunol 2017;8:1536.

9. Hamamoto N, Maemura K, Hirata I, Murano M, Sasaki S, Katsu $\mathrm{K}$. Inhibition of dextran sulphate sodium (DSS)-induced colitis in mice by intracolonically administered antibodies against adhesion molecules (endothelial leucocyte adhesion molecule-1 (ELAM-1) or intercellular adhesion molecule-1 (ICAM-1)). Clin Exp Immunol 1999;117:462-468.

10. Dieleman LA, Palmen MJ, Akol H, et al. Chronic experimental colitis induced by dextran sulphate sodium (DSS) is characterized by Th1 and Th2 cytokines. Clin Exp Immunol 1998;114:385-391.

11. Molodecky NA, Soon IS, Rabi DM, et al. Increasing incidence and prevalence of the inflammatory bowel diseases with time, based on systematic review. Gastroenterology 2012;142:46-54.

12. Round JL, Mazmanian SK. The gut microbiota shapes intestinal immune responses during health and disease. Nat Rev Immunol 2009;9:313-323.

13. Melgar S, Karlsson L, Rehnström E, et al. Validation of murine dextran sulfate sodium-induced colitis using four therapeutic 
agents for human inflammatory bowel disease. Int Immunopharmacol 2008;8:836-844.

14. Perše M, Cerar A. Dextran sodium sulphate colitis mouse model: traps and tricks. J Biomed Biotechnol 2012;2012:718617.

15. Håkansson Å, Tormo-Badia N, Baridi A, et al. Immunological alteration and changes of gut microbiota after dextran sulfate sodium (DSS) administration in mice. Clin Exp Med 2015;15:107120.

16. Barba-Vidal E, Castillejos L, Roll VF, et al. The probiotic combination of Bifidobacterium longum subsp. infantis CECT 7210 and Bifidobacterium animalis subsp. lactis BPL6 reduces pathogen loads and improves gut health of weaned piglets orally challenged with Salmonella Typhimurium. Front Microbiol 2017;8:1570.

17. Giannetti E, Staiano A. Probiotics for irritable bowel syndrome: clinical data in children. J Pediatr Gastroenterol Nutr 2016;63 Suppl 1:S25-S26.

18. van Herk EH, Te Velde AA. Treg subsets in inflammatory bowel disease and colorectal carcinoma: characteristics, role, and therapeutic targets. J Gastroenterol Hepatol 2016;31:1393-1404.

19. Park JS, Choi JW, Jhun J, et al. Lactobacillus acidophilus improves intestinal inflammation in an acute colitis mouse model by regulation of Th17 and Treg cell balance and fibrosis development. J Med Food 2018;21:215-224.

20. Izcue A, Coombes JL, Powrie F. Regulatory T cells suppress systemic and mucosal immune activation to control intestinal inflammation. Immunol Rev 2006;212:256-271.

21. Mottet C, Uhlig HH, Powrie F. Cutting edge: cure of colitis by CD4+CD25+ regulatory T cells. J Immunol 2003;170:3939-3943.

22. Canavan JB, Scottà C, Vossenkämper A, et al. Developing in vitro expanded CD45RA+ regulatory $\mathrm{T}$ cells as an adoptive cell therapy for Crohn's disease. Gut 2016;65:584-594.

23. Williams LM, Rudensky AY. Maintenance of the Foxp3-dependent developmental program in mature regulatory $\mathrm{T}$ cells requires continued expression of Foxp3. Nat Immunol 2007;8:277-284.

24. Laidlaw BJ, Cui W, Amezquita RA, et al. Production of IL-10 by $\mathrm{CD} 4(+)$ regulatory $\mathrm{T}$ cells during the resolution of infection promotes the maturation of memory CD8(+) T cells. Nat Immunol 2015;16:871-879.

25. Rennick DM, Fort MM. Lessons from genetically engineered animal models. XII. IL-10-deficient (IL-10(-/-) mice and intestinal inflammation. Am J Physiol Gastrointest Liver Physiol 2000;278:G829-G833.

26. Tilg H, van Montfrans C, van den Ende A, et al. Treatment of Crohn's disease with recombinant human interleukin 10 induces the proinflammatory cytokine interferon gamma. Gut 2002;50:191-195.

27. Kitamura H, Yamamoto S, Nakase H, et al. Role of heat shock protein 47 in intestinal fibrosis of experimental colitis. Biochem Biophys Res Commun 2011;404:599-604.

28. Chowdhury A, Fukuda R, Fukumoto S. Growth factor mRNA expression in normal colorectal mucosa and in uninvolved mucosa from ulcerative colitis patients. J Gastroenterol 1996;31:353-360.

29. Zhao HM, Huang XY, Zuo ZQ, et al. Probiotics increase T regulatory cells and reduce severity of experimental colitis in mice. World J Gastroenterol 2013;19:742-749.

30. Konieczna P, Groeger D, Ziegler M, et al. Bifidobacterium infantis 35624 administration induces Foxp3 T regulatory cells in human peripheral blood: potential role for myeloid and plasmacytoid dendritic cells. Gut 2012;61:354-366.

31. Zheng B, van Bergenhenegouwen J, Overbeek S, et al. Bifidobacterium breve attenuates murine dextran sodium sulfateinduced colitis and increases regulatory T cell responses. PLoS One 2014;9:e95441.

32. Zamani MR, Aslani S, Salmaninejad A, Javan MR, Rezaei N. PD-1/PD-L and autoimmunity: a growing relationship. Cell Immunol 2016;310:27-41.

33. Song MY, Hong CP, Park SJ, et al. Protective effects of Fc-fused PD-L1 on two different animal models of colitis. Gut 2015;64:260271.

34. Barber DL, Wherry EJ, Masopust D, et al. Restoring function in exhausted CD8 T cells during chronic viral infection. Nature 2006;439:682-687.

35. Totsuka T, Kanai T, Makita S, et al. Regulation of murine chronic colitis by CD4+CD25- programmed death- $1+\mathrm{T}$ cells. Eur J Immunol 2005;35:1773-1785.

36. Francisco LM, Salinas VH, Brown KE, et al. PD-L1 regulates the development, maintenance, and function of induced regulatory $\mathrm{T}$ cells. J Exp Med 2009;206:3015-3029. 